\title{
SPARSITY-DRIVEN MOVING TARGET DETECTION IN DISTRIBUTED MULTISTATIC FMCW RADARS
}

\author{
Gilles Monnoyer de Galland de Carnières, Thomas Feuillen, Laurent Jacques, Luc Vandendorpe
}

\author{
ICTEAM/ELEN, UCLouvain, Belgium
}

\begin{abstract}
We investigate the problem of sparse target detection from widely distributed multistatic Frequency Modulated Continuous Wave (FMCW) radar systems (using chirp modulation). Unlike previous strategies (e.g., developed for FMCW or distributed multistatic radars), we propose a generic framework that scales well in terms of computational complexity for high-resolution space-velocity grid. Our approach assumes that $(i)$ the target signal is sparse in a discrete space-velocity domain, hence allowing for non-static target detection, and (ii) the resulting multiple baseband radar signals share a common support. By simplifying the representation of the FMCW radar signals, we propose a versatile scheme balancing complexity and detection accuracy. In particular, we design a low-complexity, factorized alternative for the Matching Pursuit algorithm leveraging this simplified model, as well as an iterative methodology to compensate for the errors caused by the model simplifications. Extensive Monte-Carlo simulations of a K-band radar system show that our method achieves a fast estimation of moving target's parameters on dense grids, with controllable accuracy, and reaching state-of-the-art performances compared to previous sparsity-driven approaches.
\end{abstract}

Index Terms - Radar, sparsity, FMCW, matching pursuit, factorization.

\section{Introduction}

Monostatic and bistatic radars with a single transmitter (TX) and a single receiver (RX) only offer the possibility of locating the targets on ellipses and estimating a single component of their velocities. While the addition of antennas to form co-located Multiple Input/Multiple Output (MIMO) radars only enables the estimation of the targets' location vectors, multistatic radars with widely separated TX and RX nodes studied in this paper provide a complete estimation of all target parameters (location and velocity vectors). Moreover, multistatic radars provide spatial diversity by viewing targets from different angles, thereby enhancing the robustness of estimation [1 2 3 ] and helping target tracking [4], and recognition [5].

The target detection using radars can be formalized as the reconstruction of a sparse vector [6], i.e., with only a few non-zero elements. The support of this vector provides a profile of the targets' locations and velocities. This assumes that the sampled received signal can be decomposed with a few coefficients in a radar sensing matrix (typically a Fourier matrix for FMCW), forming a dictionary of waveforms [7, 8]. Such modeling is instrumental for the application of compressed sensing techniques to radar systems [9, 10], and for super resolution radars [11, [12]. Recent contributions on multistatic radars also formalized the target detection as the reconstruction of sparse signals [13]. Unlike co-located MIMO systems, multistatic radars do not enable phase-coherent combinations of their signals. While we could treat each received signal independently to obtain estimates of distances and radial speeds, and subsequently combine them (thanks to, e.g., triangulation [14] or trilateration [15]), this paper investigates the joint (across the received signals) modeling and estimation of the target parameters. This leads to a multiple joint reconstruction problem, coupled by a common sparsity pattern [16], therefore removes the need of an association step of multiple targets across the radars.

In [13 17, 18], an adaptation of the Matching Pursuit (MP) [19], the Block Matching Pursuit (BMP) suggested by Eldar in [20], is

GM and LJ are funded by the Belgian FNRS. used to exploit the inherent block sparsity of the received signals resulting from the unique sparsity pattern their share. In this context, the 4-D space-velocity domain is sampled into $L$ possible states. However, the value of $L$ can be tremendously large, even at the minimum sampling rate required to fully exploit the potential of the radars. For Pulse Doppler radars (PDRs), properties of the dictionary have been exploited to derive lower complexity strategies in order to overcome this issue [21].

The low power continuously emitted by FMCW radars makes them more suited for short range applications [22], such as the automotive world [23, 24, 25]. However, established dictionaries for FMCW multistatic radars only considered delay estimation in RXs; In other words, only the target localization is considered without velocity estimation [16]. The first purpose of this paper is therefore to extend this contribution by including the velocity of the targets. Our complete definition of the dictionaries enables us to derive lower complexity algorithms in a factorized fashion. To achieve this goal, we apply relevant simplifications to the complete signals model. Next, a careful analysis of the model mismatches due to the simplifications enable us to provide a bound on the estimation errors of the low complexity algorithm we derived. We then introduce an enhanced algorithm providing an iterative improvement of the target parameters' estimation, rectifying these errors while preserving the low algorithmic complexity order. The algorithms we introduce are evaluated via extensive Monte-Carlo simulations.

Notations and Conventions: Matrices and vectors are denoted by bold symbols, $[\boldsymbol{A}]_{n}$ or $\boldsymbol{A}_{n}$ is the $n$-th column of a matrix $\boldsymbol{A}$, $j=\sqrt{-1}$, and $\mathrm{c}$ is the speed of light. The scalar product between the vectors $\boldsymbol{a}$ and $\boldsymbol{b}$ reads $\langle\boldsymbol{a}, \boldsymbol{b}\rangle$. The transpose and conjugate transpose of a matrix $\boldsymbol{A}$ are $\boldsymbol{A}^{\top}$ and $\boldsymbol{A}^{H}$, respectively. The modulo operator is mod, $\circledast$ is the convolution operator, $[N]:=$ $\{1, \cdots, N\}$, and $\mathbb{C N}\left(0, \sigma^{2}\right)$ is the centered complex normal distribution of variance $\sigma^{2}$.

\section{System and signal description}

From any multistatic radar system, we acquire $Q$ distinct signals, regardless of the practical implementation. Each received signal originates from a bistatic pair of nodes, described by a TXRX pair such that RX receives, converts adequately into baseband equivalent, and samples the echo resulting from the signal transmitted by TX and reflected by the targets. For instance, a time division multiplexing between transmitters using a shared frequency band can achieve up to $Q=N_{T} \times N_{R}$, if composed of $N_{T}$ TX nodes and $N_{R}$ RX nodes. A FMCW radar transmits a signal whose waveform is described by

$$
s_{T}(t)=e^{j 2 \pi \int_{0}^{t} f_{c}\left(t^{\prime}\right) \mathrm{d} t^{\prime}}
$$

where $f_{c}(t)$ is the instantaneous carrier frequency at instant $t$. For the chirp modulated radars studied here,

$$
f_{c}(t)=f_{0}+B\left(\frac{t}{T} \bmod 1\right)
$$

where $f_{0}$ is the lower frequency, $B$ is the bandwidth of the transmitted signal and $T$ is the ramp duration.

a) Complete radar Model: We model a target as a point scatterer moving in a 2-D plane. Given $K$ targets located in $\mathcal{X}:=$ $\left\{\boldsymbol{x}_{k}\right\}_{1}^{K}$ and moving with constant velocities $\mathcal{V}:=\left\{\boldsymbol{v}_{k}\right\}_{1}^{K}$, the transmission of the $q$-th signal $(q \in[Q])$ acquired from one of the $Q$ bistatic antenna pairs follows the following channel model between the transmitted signal $s_{T}^{q}(t)$ and the received signal $s_{R}^{q}(t)$,

$$
s_{R}^{q}(t)=s_{T}^{q}(t) \circledast\left[\sum_{k \in[K]} \alpha_{k}^{q} \delta\left(t-\tau_{k}^{q}(t)\right)\right]+\varepsilon(t),
$$

where $\varepsilon(t)$ is an Additive White Gaussian Noise (AWGN), $\alpha_{k}^{q}$ are the scattering coefficients which model the effects occurring in the 
wave reflection process, including the unknown Radar Cross Sections (RCS). For the sake of simplicity, we neglect in our model the effect of clutter and direct cross talk between TXs and RXs antennas. Their study is postponed to a future work. For all $q \in[Q]$, $\tau^{q}(t)$ is a Delay-Doppler term associated to the $q$-th bistatic pair and defined by

$$
\tau_{k}^{q}(t)=\frac{1}{c}\left(r^{q}\left(\boldsymbol{x}_{k}\right)+v^{q}\left(\boldsymbol{x}_{k}, \boldsymbol{v}_{k}\right) t\right),
$$

where $r^{q}(\boldsymbol{x})$ is the bistatic range and $v^{q}(\boldsymbol{x}, \boldsymbol{v})$ is the bistatic speed of the $q$-th bistatic pair, respectively described by

$$
\begin{aligned}
r^{q}(\boldsymbol{x}) & =\left\|\boldsymbol{x}_{\mathrm{t}}^{q}-\boldsymbol{x}\right\|_{2}+\left\|\boldsymbol{x}_{\mathrm{r}}^{q}-\boldsymbol{x}\right\|_{2}, \\
v^{q}(\boldsymbol{x}, \boldsymbol{v}) & =\left\langle\frac{\boldsymbol{x}_{\mathrm{t}}^{q}-\boldsymbol{x}}{\left\|\boldsymbol{x}_{\mathrm{t}}^{q}-\boldsymbol{x}\right\|_{2}}+\frac{\boldsymbol{x}_{\mathrm{r}}^{q}-\boldsymbol{x}}{\left\|\boldsymbol{x}_{\mathrm{r}}^{q}-\boldsymbol{x}\right\|_{2}}, \boldsymbol{v}\right\rangle,
\end{aligned}
$$

where $\boldsymbol{x}_{\mathrm{t}}^{q}$ and $\boldsymbol{x}_{\mathrm{r}}^{q}$, are the respective locations of the TX and RX nodes of the $q$-th bistatic pair. After a coherent demodulation with the carrier $f_{c}(t)$ of the received signals in (3), in which we insert (2) and (1), we get the baseband signal equivalent

$$
\begin{aligned}
& e_{R}^{q}(t)=e_{\varepsilon}^{q}(t)+ \\
& \sum_{k \in[K]} \alpha_{k}^{q} e^{-j 2 \pi\left(f_{0} \tau_{k}^{q}(t)+B \tau_{k}^{q}(t)\left(\frac{t}{T} \bmod 1\right)-\frac{B}{2 T}\left(\tau_{k}^{q}(t)\right)^{2}\right),}
\end{aligned}
$$

where $e_{\varepsilon}^{q}(t)$ is the baseband equivalent of $\varepsilon(t)$. The signal is sampled at rate $T_{s}$ with $M_{s}$ samples acquired per ramp, such that $T=M_{s} T_{s}, M_{r}$ ramps are acquired. If $m_{s} \in\left[M_{s}\right]$ is the index of the sample inside a ramp and $m_{r} \in\left[M_{r}\right]$ is the index of the ramp, the sampled received signal for the $q$-th bistatic pair reads

$$
y^{q}\left[m_{s}, m_{r}\right]:=e_{R}^{q}\left(\left(m_{r}-1\right) T+m_{s} T_{s}\right) .
$$

From (4) and (7), $y^{q}$ can be decomposed as

$$
y^{q}\left[m_{s}, m_{r}\right]=\sum_{k \in[K]} \alpha_{k}^{q} d_{\boldsymbol{x}_{k}, \boldsymbol{v}_{k}}^{q}\left[m_{s}, m_{r}\right]+e_{\varepsilon}^{q}\left[m_{s}, m_{r}\right],
$$

where we define

$$
d_{\boldsymbol{x}, \boldsymbol{v}}^{q}\left[m_{s}, m_{r}\right]:=\psi_{\boldsymbol{x}, \boldsymbol{v}}^{q}\left[m_{s}\right] \phi_{\boldsymbol{x}, \boldsymbol{v}}^{q}\left[m_{r}\right] \theta_{\boldsymbol{x}, \boldsymbol{v}}^{q}\left[m_{s}, m_{r}\right],
$$

with (i) the inner signal $\psi_{\boldsymbol{x}, \boldsymbol{v}}^{q}\left[m_{s}\right]$ only depending on the ramp internal index $m_{s}$ and such that

$$
\begin{aligned}
\psi_{\boldsymbol{x}, \boldsymbol{v}}^{q}\left[m_{s}\right]:= & e^{-j 2 \pi \frac{f_{0}}{\mathrm{c}} r^{q}(\boldsymbol{x})} e^{j 2 \pi \frac{B}{2 M_{s} T_{s}} \frac{1}{c^{2}}\left(r^{q}(\boldsymbol{x})\right)^{2}} \\
& e^{-j 2 \pi \frac{1}{c}\left(\frac{B}{M_{s}} r^{q}(\boldsymbol{x})+f_{0} T_{s} v^{q}(\boldsymbol{x}, \boldsymbol{v})\right) m_{s}},
\end{aligned}
$$

(ii) the outer signal $\phi_{\boldsymbol{x}, \boldsymbol{v}}^{q}\left[m_{r}\right]$ defined by,

$$
\phi_{\boldsymbol{x}, \boldsymbol{v}}^{q}\left[m_{r}\right]:=e^{-j 2 \pi \frac{f_{0}}{c} T v^{q}(\boldsymbol{x}, \boldsymbol{v}) m_{r}},
$$

and (iii) the coupling signal $\theta_{\boldsymbol{x}, \boldsymbol{v}}^{q}\left[m_{s}, m_{r}\right]$, combining both $m_{s}$ and $m_{r}$ in

$$
\begin{aligned}
\theta_{\boldsymbol{x}, \boldsymbol{v}}^{q}\left[m_{s}, m_{r}\right]:= & e^{-j 2 \pi \frac{1}{\mathrm{c}} \frac{B}{M_{s}} v^{q}(\boldsymbol{x}, \boldsymbol{v})\left(m_{r} T+m_{s} T s\right) m_{s}} \\
& e^{j 2 \pi \frac{B}{M_{s} T_{s}} \frac{1}{c^{2}}\left(r^{q}(\boldsymbol{x}) v^{q}(\boldsymbol{x}, \boldsymbol{v})\left(m_{r} T+m_{s} T_{s}\right)\right)} \\
& e^{j 2 \pi \frac{B}{2 M_{s} T_{s}} \frac{1}{c^{2}}\left(v^{q}(\boldsymbol{x}, \boldsymbol{v})\left(m_{r} T+m_{s} T_{s}\right)\right)^{2}} .
\end{aligned}
$$

Note that we have arbitrarily gathered in $\psi_{\boldsymbol{x}, \boldsymbol{v}}^{q}\left[m_{s}\right]$ the factors independent of both $m_{s}$ and $m_{r}$.

b) Joint-sparse model of radar measurements: We define a joint acquisition model where all bistatic pairs simultaneously observe a common scene composed of a few moving targets. We assume them to be localized on a given (separable) space-velocity grid with no more than one target per grid cell, i.e., $\mathcal{X} \subset \Omega_{x}:=$ $\left\{\boldsymbol{\omega}_{n}^{x}\right\}_{n=1}^{N_{x}}$ and $\mathcal{V} \subset \Omega_{v}:=\left\{\boldsymbol{\omega}_{\dot{n}}^{v}\right\}_{\dot{n}=1}^{N_{v}}$, for some location grid $\Omega_{x} \subset \mathbb{R}^{2}$ and velocity grid $\Omega_{v} \subset \mathbb{R}^{2}$ made of $N_{x}$ locations and $N_{v}$ velocities, respectively. We postpone to a future study the more involved case of off-grid targets, as studied in [26] for MIMO radars. From this assumption, (8) can be recast for each $q \in[Q]$ as the decomposition of $\boldsymbol{y}^{q}:=\left(y^{q}[1,1], \cdots, y^{q}\left[M_{s}, M_{r}\right]\right)^{\top}$ in the dictionary $\boldsymbol{D}^{q}$ of the $q$-th bistatic pair, where

$$
\boldsymbol{D}^{q}:=\left[\boldsymbol{d}_{1,1}^{q}, \boldsymbol{d}_{1,2}^{q}, \cdots, \boldsymbol{d}_{N_{x}, N_{v}}^{q}\right] \in \mathbb{C}^{M_{s} M_{r} \times N_{x} N_{v}} .
$$

This dictionary is composed of the $N_{x} N_{v}$ atoms defined by

$$
\boldsymbol{d}_{n, \dot{n}}^{q}:=\left(d_{\boldsymbol{\omega}_{n}^{x}, \boldsymbol{\omega}_{\dot{n}}^{v}}^{q}[1,1], d_{\boldsymbol{\omega}_{n}^{x}, \boldsymbol{\omega}_{\dot{n}}^{v}}^{q}[1,2], \cdots, d_{\boldsymbol{\omega}_{n}^{x}, \boldsymbol{\omega}_{\dot{n}}^{v}}^{q}\left[M_{s}, M_{r}\right]\right)^{\top},
$$

where $d_{n, \dot{n}}^{q}$ is the $q$-th dictionary's atom corresponding to the $n$-th location and $\dot{n}$-th velocity (with $n \in\left[N_{x}\right]$ and $\dot{n} \in\left[N_{v}\right]$,).

We can, then, turn (8) into the model

$$
\boldsymbol{y}^{q}=\boldsymbol{D}^{q} \boldsymbol{s}^{q}+\boldsymbol{\varepsilon}^{q} \in \mathbb{C}^{M_{s} M_{r}}
$$

where $\varepsilon^{q}$ is the AWGN of the $q$-th received signal. The $K$-sparse target vector $\boldsymbol{s}^{q}:=\left(s^{q}[1,1], \cdots, s^{q}\left[N_{x}, N_{v}\right]\right)^{\top}$ is defined from

$$
s^{q}[n, \dot{n}]= \begin{cases}\alpha_{k}^{q} & \text { if }\left(\boldsymbol{x}_{k}, \boldsymbol{v}_{k}\right)=\left(\boldsymbol{\omega}_{n}^{x}, \boldsymbol{\omega}_{\dot{n}}^{v}\right) \\ 0 & \text { otherwise. }\end{cases}
$$

The $Q$ vectors $\left\{\boldsymbol{s}^{q}\right\}_{q=1}^{Q}$ thus share a common support, i.e., $\operatorname{supp} \boldsymbol{s}^{q}=\mathcal{S}$ for all $q \in[Q]$, with $\mathcal{S} \subset\left[N_{x} N_{v}\right]$ and $|S| \leq K$.

c) Simplified radar model: While the structure of the dictionaries in (13) is rather intricate, a simplified joint sparse model can be derived from a few simplifications made on the signals and system properties: (S1) the transmitted signal is narrowband, i.e., $B \ll f_{0}$; (S2) for all $k \in[K], q \in[Q]$, we have $\tau_{k}^{q}<T_{s}$ such that the echo of transmitted a ramp is acquired when its TX is still emitting the same ramp, hence $r^{q}\left(\boldsymbol{x}_{k}\right)<c T_{s} ;(S 3) \Omega_{v}$ is chosen unambiguously, such that the sampling frequency $1 / T$ between ramps which samples $\phi_{\boldsymbol{x}, \boldsymbol{v}}^{q}\left[m_{r}\right]$ fulfills the Shannon-Nyquist sampling theorem. This means that $|v(\boldsymbol{x}, \boldsymbol{v})| \leq 2\|\boldsymbol{v}\|_{2} \leq \frac{c}{2 f_{0} T} \forall \boldsymbol{v} \in \Omega_{v}$. These three simplifications lead to the conclusion that the coupling signal in (12) respects $\theta_{\boldsymbol{x}, \boldsymbol{v}}\left[m_{s}, m_{r}\right] \simeq 1$. Moreover, (S3) ensures that the velocity has small impact on the inner signal and

$$
\psi_{\boldsymbol{x}, \boldsymbol{v}}^{q}\left[m_{s}\right] \simeq \psi_{\boldsymbol{x}}^{q}\left[m_{s}\right]:=\psi_{\boldsymbol{x}, \mathbf{0}}^{q}\left[m_{s}\right]
$$

In practice this approximation amounts to neglecting the Doppler effect within each ramp and thus only considering it between the consecutive ramps. It is equivalent in the context of (8) to set $\boldsymbol{v}=0$ in $\psi_{\boldsymbol{x}, \boldsymbol{v}}^{q}$. This simplification is known to generate distorsions, as studied in 27, 28, 29], but often enables the use of 2D-Discrete Fourier Transforms (DFT) for monostatic FMCW radars. However, the shared grids used to establish the joint model of the multistatic radar signals preclude the use of 2D-DFT. Still, the simplification in (15) enables an analog factorization of the model.

Together, these simplifications bring this simplified multistatic radar model:

$$
y^{q}\left[m_{s}, m_{r}\right] \simeq \sum_{k \in[K]} \alpha_{k}^{q} \psi_{\boldsymbol{x}_{k}}^{q}\left[m_{s}\right] \phi_{\boldsymbol{x}_{k}, \boldsymbol{v}_{k}}^{q}\left[m_{r}\right]+e_{\varepsilon}^{q}\left[m_{s}, m_{r}\right] .
$$

Interestingly, we can recast 16 as a factorized joint model for multistatic FMCW radars by defining the $q$-th measurement matrix $\boldsymbol{Y}^{q} \in \mathbb{C}^{M_{s} \times M_{r}}$ and target matrix $\boldsymbol{S}^{q} \in \mathbb{C}^{N_{x} \times N_{v}}$ as $\left(\boldsymbol{Y}^{q}\right)_{m_{s}, m_{r}}:=y^{q}\left[m_{s}, m_{r}\right]$, and $\left(\boldsymbol{S}^{q}\right)_{n, \dot{n}}:=s^{q}[n, \dot{n}]$, for all $m_{s} \in\left[M_{s}\right], m_{r} \in\left[M_{r}\right], n \in\left[N_{x}\right], \dot{n} \in\left[N_{v}\right], q \in[Q]$. The noise matrices $\boldsymbol{E}^{q} \in \mathbb{C}^{N_{x} \times N_{v}}$ are defined similarly from $e_{\varepsilon}^{q}$. We also introduce the inner dictionary $\boldsymbol{\Psi}^{q} \in \mathbb{C}^{M_{s} \times N_{x}}$ and outer dictionary $\boldsymbol{\Phi}_{n}^{q} \in \mathbb{C}^{M_{r} \times N_{v}}$ such that $\left(\boldsymbol{\Psi}^{q}\right)_{m_{s}, n}:=\psi_{\boldsymbol{\omega}_{n}^{x}}\left[m_{s}\right]$, and $\left(\boldsymbol{\Phi}_{n}^{q}\right)_{m_{r}, \dot{n}}:=\phi_{\boldsymbol{\omega}_{n}^{x}, \omega_{\dot{n}}^{v}}\left[m_{r}\right]$, for all $m_{s} \in\left[M_{s}\right], m_{r} \in\left[M_{r}\right], n \in$ $N_{x}, \dot{n} \in N_{v}, q \in[Q]^{n}$.

In this setting, the matrix form of 16 is "factorized" as

$$
\begin{aligned}
& \boldsymbol{Y}^{q} \simeq \boldsymbol{\Psi}^{q} \boldsymbol{P}^{q}+\boldsymbol{E}^{q}, \\
& \boldsymbol{P}^{q}=\left[\boldsymbol{\Phi}_{1}^{q}\left[\boldsymbol{S}^{q \top}\right]_{1}, \cdots, \boldsymbol{\Phi}_{N_{x}}^{q}\left[\boldsymbol{S}^{q \top}\right]_{N_{x}}\right]^{\top} .
\end{aligned}
$$

Note that, from (14), $\boldsymbol{S}^{q}$ has no more than $K$ non-zero rows and columns. Therefore, from 17b, the matrices $\boldsymbol{P}^{q}$ have less than $K$ non-zero columns, i.e., they are row-sparse.

\section{Signal recovery algorithms}

The field of computational harmonic analysis provides many algorithms to sparsely decompose a signal in a given dictionary, e.g., Basis Pursuit (BP) [30], Matching Pursuit (MP) [19], and MP improvements such as Orthogonal Matching Pursuit (OMP) [31, 32]. To take advantage of the shared sparsity pattern of the vectors $\boldsymbol{s}^{q}, q \in[Q]$ in $(13)$, we can use the Block Matching Pursuit (BMP) [20] that is well suited for multistatic radar applications (see below). As BMP is not adapted to dense grids [21], we introduce the Factorized Block Matching Pursuit (FBMP), suited for partially factorized models such as (16, an the Iterative-FBMP (IFBMP) that iteratively corrects the errors committed by FBMP. 


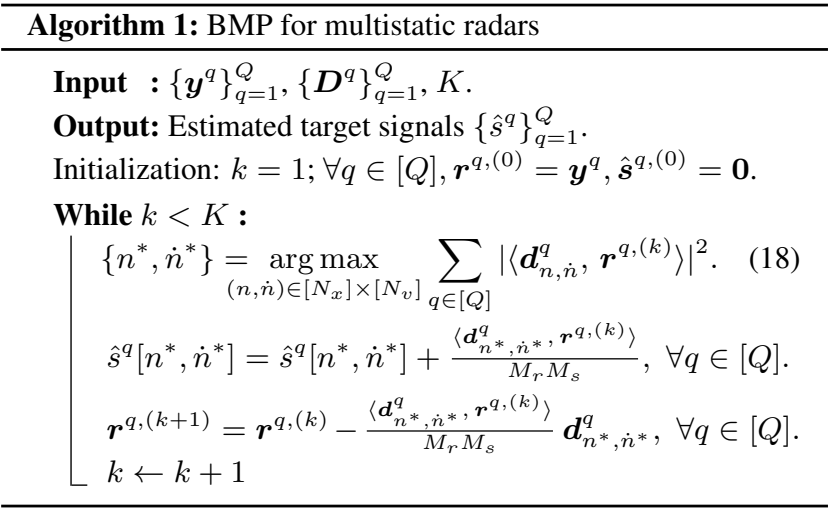

a) Block Matching Pursuit: Alg. 1 directly formulates BMP to the joint multistatic radars model 8). BMP proceeds by jointly processing the $Q$ bistatic TX-RX pairs: at each iteration, BMP first selects in 18 the indices $\left\{n^{*}, \dot{n}^{*}\right\}$ corresponding to the atoms collaboratively maximizing their correlation with the $Q$ residuals $\boldsymbol{r}^{q,(k)}$ (each set to $\boldsymbol{y}^{q}$ at $k=0$ ), stores these correlations in $\hat{s}^{q}$, and finally updates these residuals by removing the contribution of the selected atoms. The computational complexity of BMP is dominated by 18 as this step requires us to compute the contributions of all location-velocity indices to pick the largest one. Overall, the complexity scales like $O\left(K Q M_{r} M_{s} N_{x} N_{v}\right)$ at each iteration, which becomes quickly unaffordable for dense grids $\Omega_{x}$ and $\Omega_{v}$.

b) Factorized BMP: We propose a new algorithm, the Factorized Block Matching Pursuit (FBMP), which reduces the complexity of BMP by leveraging the factorized model $(17)$, i.e., by replacing 18 in Alg. 1 by an approximate method. Writing $\boldsymbol{\psi}_{n}^{q}$ (and $\phi_{n, \dot{n}}^{q}$ ) for the $n$-th (resp. $\dot{n}$-th) column of $\boldsymbol{\Psi}^{q}$ (resp. $\boldsymbol{\Phi}_{n}^{q}$ ), and given the residuals $\boldsymbol{R}^{q,(k)}=\left(\boldsymbol{r}_{1}^{q,(k)}, \cdots, \boldsymbol{r}_{M_{r}}^{q,(k)}\right) \in \mathbb{C}^{N_{x} \times M_{r}}$ initialized to $\boldsymbol{R}^{q,(0)}=\boldsymbol{Y}^{q}$ for $q \in[Q]$, we perform at each FBMP iteration the following two-step procedure. First, inspired from [17a, the location index $n^{*}$ is computed from the inner dictionaries $\mathbf{\Psi}^{q}$, independently of $\dot{n}^{*}$, in other words $n^{*}$ is the location index of the inner atoms collaboratively maximizing their "intra-ramp" correlation with all the residuals, $n^{*}$ is computed by

$$
n^{*}=\arg \max _{n \in\left[N_{x}\right]} \sum_{q \in[Q]} \sum_{m_{r} \in\left[M_{r}\right]}\left|\left\langle\boldsymbol{\psi}_{n}^{q}, \boldsymbol{r}_{m_{r}}^{q,(k)}\right\rangle\right|^{2} .
$$

Next, by considering $17 \mathrm{~b}$, we find $\dot{n}^{*}$ from the outer dictionaries $\boldsymbol{\Phi}_{n^{*}}^{q}$ defined on $n^{*}$ for each $q \in[Q]: \dot{n}^{*}$ is the velocity index corresponding to the outer atoms localized on $n^{*}$ that collaboratively maximize their correlation with $\boldsymbol{p}_{n^{*}}^{q}$,

$$
\dot{n}^{*}=\arg \max _{\dot{n} \in\left[M_{r}\right]} \sum_{q \in[Q]}\left|\left\langle\phi_{n^{*}, \dot{n}}^{q}, \tilde{\boldsymbol{p}}_{n^{*}}^{q}\right\rangle\right|^{2} .
$$

with $\boldsymbol{p}_{n^{*}}^{q}:=\left(\left\langle\boldsymbol{\psi}_{n^{*}}^{q}, \boldsymbol{r}_{1}^{q,(k)}\right\rangle, \cdots,\left\langle\boldsymbol{\psi}_{n^{*}}^{q}, \boldsymbol{r}_{M_{r}}^{q,(k)}\right\rangle\right)^{\top}$.

The computational complexity of this two-step procedure reduces to $O\left(K Q\left(M_{r} M_{s} N_{x}+M_{s} N_{v}\right)\right)$, which is lower than for 18$]$ in BMP for dense grids. However, FBMP suffers from the model mismatches induced by the simplifications introduced in Sec. 2, resulting in a wrong estimation of the targets' locations.

In fact, in noiseless condition, neglecting the influence of the coupling signals, and in the context of a single target $(K=1)$ with parameters $(\boldsymbol{x}, \boldsymbol{v})$ where $\boldsymbol{v}$ is known (in an oracle context), the target location would be more accurately estimated by 19$]$ if the atoms of the inner dictionaries $\boldsymbol{\Psi}_{\boldsymbol{v}}^{q}$ such that $\left(\boldsymbol{\Psi}_{\boldsymbol{v}}^{q}\right)_{m_{s}, n}:=$ $\psi_{\boldsymbol{\omega}_{n}^{x}, \boldsymbol{v}}\left[m_{s}\right]$ were used instead of the atoms $\boldsymbol{\psi}_{n}^{q}$ of $\boldsymbol{\Psi}^{q}=\boldsymbol{\Psi}_{\mathbf{0}}^{q}$. However, Thm. 1 shows that if $\Omega_{v}$ respects a Shannon-Nyquist criterion, and if the TX and RX nodes are located sufficiently far of the location grid, then for any $q \in[Q]$ and $\boldsymbol{x} \in \Omega_{x}$, the atom associated with the location $\boldsymbol{x}$ in the dictionary $\boldsymbol{\Psi}_{v}^{q}$ tends to be proportional to the atom associated to a location $\tilde{\boldsymbol{x}}$ shifted in the direction of $\boldsymbol{v}$ in the dictionary $\boldsymbol{\Psi}^{q}$ (assuming $\tilde{\boldsymbol{x}}$ is still in $\Omega_{x}$ ). Hence, for a sufficiently dense location grid, the dictionaries $\boldsymbol{\Psi}^{q}, q \in[Q]$ approximate the dictionaries $\boldsymbol{\Psi}_{\boldsymbol{v}}^{q}, q \in[Q]$ with the atoms' indices shifted in a manner that depends on $\boldsymbol{v}$ (see also Sec. 4 .
Theorem 1. Given some $\lambda>3, \gamma:=\frac{f_{0} M_{s} T_{s}}{B}$, and the 2-D positions $\boldsymbol{x}_{\mathrm{t}}^{q}$ and $\boldsymbol{x}_{\mathrm{r}}^{q}$ of the TX and RX antennas for each bistatic pair $q \in[Q]$, respectively, if

$$
\begin{gathered}
\max _{\boldsymbol{v} \in \Omega_{v}}|v(\boldsymbol{x}, \boldsymbol{v})|_{2} \leq 2 \max _{\boldsymbol{v} \in \Omega_{v}}\|\boldsymbol{v}\|_{2} \leq \frac{\mathrm{c}}{2 f_{0} T}, \\
\min _{\boldsymbol{x} \in \Omega_{x}, q \in[Q]} \min \left(\left\|\boldsymbol{x}_{\mathrm{t}}^{q}-\boldsymbol{x}\right\|_{2},\left\|\boldsymbol{x}_{\mathrm{r}}^{q}-\boldsymbol{x}\right\|_{2}\right)>\lambda \frac{\mathrm{c}}{4 B},
\end{gathered}
$$

then, there exist a function $A^{q}(\boldsymbol{x}, \boldsymbol{v}) \in \mathbb{C}$ with $\left|A^{q}(\boldsymbol{x}, \boldsymbol{v})\right|=1$, independent of $m_{s}$, such that, for all $m_{s} \in\left[M_{s}\right]$ and $q \in[Q]$,

$$
\left|\psi_{\boldsymbol{x}, \boldsymbol{v}}^{q}\left[m_{s}\right]-A^{q}(\boldsymbol{x}, \boldsymbol{v}) \psi_{\tilde{\boldsymbol{x}}, \mathbf{0}}^{q}\left[m_{s}\right]\right|=O\left(\frac{1}{\lambda}\right),
$$

where $\tilde{\boldsymbol{x}}:=\boldsymbol{x}+\gamma \boldsymbol{v}$ and $\|\tilde{\boldsymbol{x}}-\boldsymbol{x}\|_{\infty} \leq \frac{\mathrm{c}}{4 \sqrt{2} B}$.

Because we always have $\left|A^{q}(\boldsymbol{x}, \boldsymbol{v})\right|=1$ the above reasoning implies that, for a highly dense location grid, FBMP tends to estimate $\hat{\boldsymbol{x}}=\boldsymbol{\omega}_{n}^{x}=\tilde{\boldsymbol{x}}$ instead of $\boldsymbol{x}$. Moreover, the bound on the estimation error $\|\tilde{\boldsymbol{x}}-\boldsymbol{x}\|_{\infty}$ provided by Thm. 1 asserts that, for a sufficiently large value of $\lambda$, the estimated location $\hat{\boldsymbol{x}}$ is close to the exact target's location $\boldsymbol{x}$ compared to the distance between the target and the TX and RX nodes. This implies that the scalar product in (6), and hence the chosen outer dictionaries for the velocity estimation, $\boldsymbol{\Phi}_{n}^{q}, q \in[Q]$, are only poorly affected by the location estimation error. Therefore, $\hat{\boldsymbol{v}}=\boldsymbol{\omega}_{\dot{n}^{*}}^{v}$ is also close to the exact target's velocity $\boldsymbol{v}$ when compared to the initial arbitrary guess $\hat{\boldsymbol{v}}=0$. c) Iterative FBMP (IFBMP): We can compensate the errors caused by the model mismatch committed by FBMP by iteratively improving the space-velocity indices of each selected atom, and this with only a slight increase in computational complexity.

IFBMP is similar to FBMP except that at the $k$-th iteration, the selection step is replaced by $N_{\mathrm{it}}$ successive improvements of the atom space-velocity parameters according to a sequence of indices $\left\{\left(n^{i}, \dot{n}^{i}\right): 0 \leq i \leq N_{\mathrm{it}}\right\}$. A similar strategy was proposed in [33] to improve MP in the selection of atoms in a dictionary made of Gaussian chirps.

Following the notations introduced for FBMP, at initialization, $\left(n^{0}, \dot{n}^{0}\right)$ are found from (19) and 20). Next, for $i \geq 1$, the $i$-th space-velocity improvement $\left(n^{i}, \dot{n}^{i}\right)$ is obtained from

$$
\begin{aligned}
& n^{i}=\underset{n \in\left[N_{x}\right]}{\arg \max } \sum_{q \in[Q]} \sum_{m_{r} \in\left[M_{r}\right]}\left|\left\langle\boldsymbol{\psi}_{n, \dot{n}^{i-1}}^{q}, \boldsymbol{r}_{m_{r}}^{q,(k)}\right\rangle\right|^{2}, \\
& \dot{n}^{i}=\underset{\dot{n} \in\left[M_{r}\right]}{\arg \max } \sum_{q \in[Q]}\left|\left\langle\boldsymbol{\phi}_{n^{i}, \dot{n}}^{q}, \tilde{\boldsymbol{p}}_{i}^{q}\right\rangle\right|^{2},
\end{aligned}
$$

with $\boldsymbol{p}_{i}^{q}:=\left(\left\langle\boldsymbol{\psi}_{n^{i}, \dot{n}^{i-1}}^{q}, \boldsymbol{r}_{1}^{q,(k)}\right\rangle, \cdots,\left\langle\boldsymbol{\psi}_{n^{i}, \dot{n}^{i-1}}^{q}, \boldsymbol{r}_{M_{r}}^{q,(k)}\right\rangle\right)^{\top}$, and $\boldsymbol{\psi}_{n, \dot{n}}^{q}:=\left(\psi_{\boldsymbol{\omega}_{n}^{x}, \boldsymbol{\omega}_{\dot{n}}^{v}}^{q}[1], \cdots, \psi_{\boldsymbol{\omega}_{n}^{x}, \boldsymbol{\omega}_{\dot{n}}^{v}}^{q}\left[M_{s}\right]\right)^{\top}$ for $n \in\left[N_{x}\right], \dot{n} \in$ $\left[N_{v}\right]$, and $q \in[Q]$. Finally, $\left(n^{*}, \dot{n}^{*}\right)=\left(n^{N_{\mathrm{it}}}, \dot{n}^{N_{\mathrm{it}}}\right)$ are the returned indices of the $k$-th selected atom of IFBMP.

The complexity of IFBMP scales like $O\left(K N_{\mathrm{it}} Q\left(M_{r} M_{s} N_{x}+\right.\right.$ $\left.M_{s} N_{v}\right)$ ). The improvement brought by IFBMP over FBMP, as well as its convergence, strongly depends on the location of the TX and RX antennas and the geometry of the grids $\Omega_{x}$ and $\Omega_{v}$; their optimization is postponed to a future study.

\section{Monte-Carlo evaluation}

The three algorithms BMP, FBMP and IFBMP share the three last steps of Alg. 1 but differ in the computation of $\left\{n^{*}, \dot{n}^{*}\right\}$ at each iteration. Because BMP uses the complete exact model, we expect it to exhibit the best performance with accurate reconstruction in noiseless condition if $K=1$. Both FBMP and IFBMP provide approximate estimations of $\left\{n^{*}, \dot{n}^{*}\right\}$ but they shorten the computing time. The first of the simulation highlights the estimation errors of FBMP caused by the model mismatch and the correction brought by IFBMP. They are therefore performed with $K=1$ in noiseless conditions. We also assumed $\alpha_{k}^{q} \stackrel{\text { i.i.d. }}{\sim} \mathbb{C N}(0,1), q \in[Q], k \in[K]$. a) Simulated radar System: The location of the antennas and the grids' properties are parameters that can be optimized according to different criterion [34, 35] but are arbitrarily chosen in this work. We simulated a multistatic FMCW radar system composed of 2 TX nodes located in $\boldsymbol{x}_{T X}^{(1)}=(0,-2.5), \boldsymbol{x}_{T X}^{(2)}=(7.5,-10)$ 

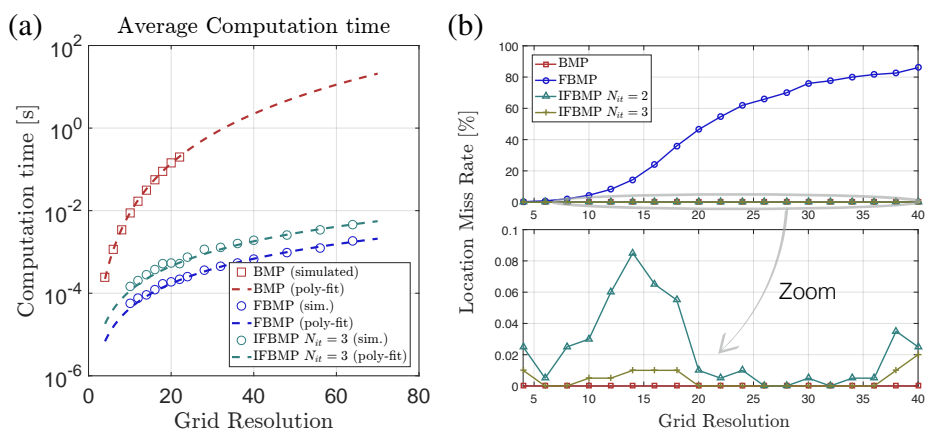

(c)

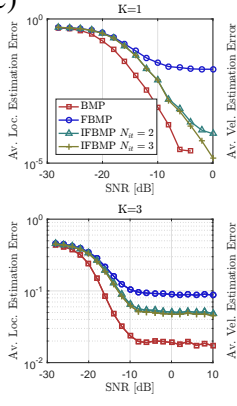

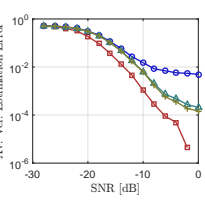

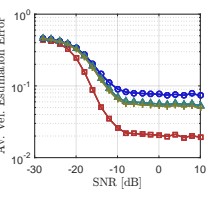

(d)

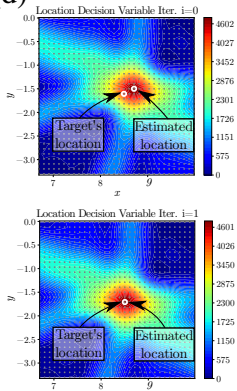

Fig. 1: (a) Average computation time of BMP and (I)FMP in function of the grid resolution. The polynomial fit of the BMP comp. time is $8.6810^{-7} \xi^{4} \mathrm{~s}$. The poly-fit of the FBMP comp. time is $4.2810^{-7} \xi^{2} \mathrm{~s}$. The poly-fit of the IFBMP comp. time is $1.1210^{-6} \xi^{2} \mathrm{~s}$. (b) Location Miss Rate vs the grid resolution for a single target, for an increasing number of iterative corrections. (c) Evolution of the average estimation error (right: location, left: velocity) with respect to the SNR with $K=1$ (top), $K=3$ (bottom). (d) Example of mapping of the decision variable for the location estimation used with IFBMP, for $K=1$ when $i=0$ (top) and $i=1$ (bottom).

and 2 RX nodes located in $\boldsymbol{x}_{R X}^{(1)}=(0,2.5), \boldsymbol{x}_{R X}^{(2)}=(12.5,-10)$, constituting $Q=4$ bistatic pairs. The bandwidth of the signals is $B=250 \mathrm{MHz}$ and the lower carrier frequency is $f_{0}=24 \mathrm{GHz}$ (K-band). Each bistatic pair provides a sampled signal with $M_{r}=$ $M_{s}=16$ at a sampling rate $\frac{1}{T_{s}}=50 \mathrm{kHz}$. The grids, arbitrarily chosen squared and uniformly sampled, are defined unambiguously according to Shannon-Nyquist: $\Omega_{v}$ defines a square centered in $\boldsymbol{v}=\mathbf{0}$ of length $L_{v}=\frac{c}{2 \sqrt{2} f_{0} T}$, which matches 21]; and $\Omega_{x}$ defines a square of length $L_{x}=M_{s} \frac{\mathrm{c}}{2 \sqrt{2} B}$ with its bottom left corner in $\boldsymbol{x}=(5,-5)$, which matches the more general formulation of the Shannon-Nyquist sampling theorem with the frequency band non-centered in 0 , fulfilled by the sufficient condition $\left|\max _{\boldsymbol{x} \in \Omega_{x}} d_{b}^{q}(\boldsymbol{x})-\min _{\boldsymbol{x} \in \Omega_{x}} d_{b}^{q}(\boldsymbol{x})\right| \leq M_{s} \frac{c}{B}$ for all $q \in[Q]$. The default sampling of these square grids are respectively $M_{s} \times M_{s}$ and $M_{r} \times M_{r}$, thus $N_{x}=M_{s}^{2}$ and $N_{v}=M_{r}^{2}$.

b) Algorithms Evaluation: To evaluate estimation errors caused by the mismatch and validate the lower computational cost promised by our algorithms, we swept the value of the grid resolution $\xi=\sqrt{N_{x}}=\sqrt{N_{v}}$. For each grid resolution, we simulated 20,000 times a target randomly selected on the current grid, and estimated its parameters using BMP and (I)FBMP. Fig. 11.a) confirms that the average computation times to compute $\left\{n^{*}, \dot{n}^{*}\right\}$ fit the complexities $O\left(\xi^{2}\right)$ and $O\left(\xi^{4}\right)$ respectively expected for, (I)FBMP and BMP. Fig. 1. b) confirms that, in noiseless condition BMP makes no estimation error, while the miss rate of FBMP the rate of wrong cell selection in the grids - grows toward almost $100 \%$ when the grid gets very dense because of the shift described in Thm. 1. which is more often accounted for with dense grids. The average location estimation error grows from $0 \%$ to $2.5 \%$ when $\xi \leq 16$, then remains constant when $\xi>16$. In short, lowdensity grids lead to few but large errors while high-density grids lead to small but systematic errors. The zoomed figure (Fig. 11 b)) shows that the location errors of FBMP are almost completely compensated for within a very small number of iterations with IFBMP. An example of detection of a target in $\boldsymbol{x}=(8.5,-1.7)$ and $\boldsymbol{v}=(6,6)$ using two iterations of IFBMP is provided in Fig. 11 d). The robustness against noise is evaluated from an operating point set to $\xi=16$. Figure 1 (c) shows the evolution of the average location and velocity estimation errors (LEE and VEE, respectively), defined from the parameters estimates $\hat{\boldsymbol{x}}_{k}$ and $\hat{\boldsymbol{v}}_{k}, k \in[K]$ as

LEE $:=\frac{1}{K} \sum_{k \in[K]} \frac{\left\|\hat{\boldsymbol{x}}_{k}-\boldsymbol{x}_{k}\right\|_{2}}{L_{x}}, \mathrm{VEE}:=\frac{1}{K} \sum_{k \in[K]} \frac{\left\|\hat{\boldsymbol{v}}_{k}-\boldsymbol{v}_{k}\right\|_{2}}{L_{v}}$,

in function of the signal to noise ratio (SNR), defined from $\sigma_{e}^{2}$, the common noise power for all bistatic pair, as

$$
\mathrm{SNR}=\frac{1}{K} \frac{\mathbb{E}\left(\sum_{q \in[Q]}\left\|\boldsymbol{D}^{q} \boldsymbol{s}^{q}\right\|_{2}^{2}\right)}{\mathbb{E}\left(\sum_{q \in[Q]}\left\|\boldsymbol{e}^{q}\right\|_{2}^{2}\right)}=\frac{1}{\sigma_{e}^{2}} .
$$

IFBMP exhibits a lower robustness against noise than BMP as a result of the non-coherent summation performed in (19). Moreover, when $K>1$, IFBMP goes toward a higher error value than BMP when the SNR goes to infinity. The main effect occurring here is the detection of ghost targets [36].
In a nutshell, our simulations confirm that IFBMP, that we proposed as an alternative to BMP, provides the same quality of target parameters estimations as BMP in noiseless condition if $K=1$ while being faster and practical for dense grids. Yet, its smaller complexity comes at the price of a lower robustness against noise.

\section{Conclusions and perspectives}

While algorithms for sparse signals reconstruction have gained in popularity in target detection with multistatic radars, the proposed adaptations such as the Block Matching Pursuit are computationally not scalable. We have shown how an explicit formulation of the dictionaries obtained from FMCW chirp-modulated signals enable simplifications and methods for dramatically reducing this complexity with limited losses of precision, as shown by Thm 1 The comparison of BMP with (I)FBMP we introduced confirmed the lower computational cost of our methods and showed that the precision losses are compensated while keeping identical computational complexity when using IFBMP. Therefore, this work is expected to allow affordable use of sparse reconstruction for FMCW multistatic radars and further lead to the design of multistatic FMCW compressive radars. Yet, the overall geometry of both the radar system and the grids provide degrees of freedom to be optimized with the help of the derivation of theoretical bounds such as Cramer-Rao bounds or Ziv-Zakai bounds.

\section{A Proof of Theorem 1}

Considering (10), the only factor of $\psi_{\boldsymbol{x}, \boldsymbol{v}}^{q}\left[m_{s}\right]$ that depends on $m_{s}$ is $\rho_{\boldsymbol{x}, \boldsymbol{v}}^{q}\left[m_{s}\right]:=\exp \left(-j \frac{2 \pi}{c}\left(\frac{B}{M_{s}} r^{q}(\boldsymbol{x})+f_{0} T_{s} v^{q}(\boldsymbol{x}, \boldsymbol{v})\right) m_{s}\right)$. We must thus prove that $\left|\rho_{\boldsymbol{x}, \boldsymbol{v}}^{q}\left[m_{s}\right]-\rho_{\tilde{\boldsymbol{x}}, \mathbf{0}}^{q}\left[m_{s}\right]\right|=O\left(\frac{1}{\lambda}\right)$.

We first note that $\left\|\boldsymbol{x}_{\mathrm{t}}^{q}-\tilde{\boldsymbol{x}}\right\|_{2}=\left\|\boldsymbol{x}_{\mathrm{t}}^{q}-\boldsymbol{x}\right\|_{2} \sqrt{1+\kappa}$ with $\kappa:=$ $2 \gamma\left\langle\boldsymbol{x}_{\mathrm{t}}^{q}-\boldsymbol{x}, \boldsymbol{v}\right\rangle\left\|\boldsymbol{x}_{\mathrm{t}}^{q}-\boldsymbol{x}\right\|_{2}^{-2}+\gamma^{2}\|\boldsymbol{v}\|_{2}^{2}\left\|\boldsymbol{x}_{\mathrm{t}}^{q}-\boldsymbol{x}\right\|_{2}^{-2}$. Using 21, $T=M_{s} T_{s}$, and 22, we find $\gamma\|\boldsymbol{v}\|_{2} \leq \frac{c}{4 B}<\frac{1}{\lambda}\left\|\boldsymbol{x}_{\mathrm{t}}^{q}-\boldsymbol{x}\right\|_{2}$.

Therefore, we show easily that $\kappa \geq \lambda^{-2}-2 \lambda^{-1} \geq-1$ and $\kappa \leq 3 \lambda^{-1}$, and since $1+\frac{s}{2}(1-s) \leq \sqrt{1+s} \leq 1+\frac{s}{2}$ for all $s \geq-1$ and $\gamma^{2} \frac{\|\boldsymbol{v}\|_{2}^{2}}{\left\|\boldsymbol{x}_{\mathrm{t}}^{q}-\boldsymbol{x}\right\|_{2}} \leq \frac{\gamma\|\boldsymbol{v}\|_{2}}{\lambda},\left\|\boldsymbol{x}_{\mathrm{t}}^{q}-\tilde{\boldsymbol{x}}\right\|_{2}$ is smaller than

$$
\left\|\boldsymbol{x}_{\mathrm{t}}^{q}-\boldsymbol{x}\right\|_{2}\left(1+\frac{\kappa}{2}\right) \leq\left\|\boldsymbol{x}_{\mathrm{t}}^{q}-\boldsymbol{x}\right\|_{2}+\gamma \frac{\left\langle\boldsymbol{x}_{\mathrm{t}}^{q}-\boldsymbol{x}, \boldsymbol{v}\right\rangle}{\left\|\boldsymbol{x}_{\mathrm{t}}^{q}-\boldsymbol{x}\right\|_{2}}+\frac{\gamma\|\boldsymbol{v}\|_{2}}{2 \lambda},
$$
and larger than

$$
\begin{array}{r}
\left\|\boldsymbol{x}_{\mathrm{t}}^{q}-\boldsymbol{x}\right\|_{2}\left(1+\frac{\kappa}{2}(1-\kappa)\right) \geq\left\|\boldsymbol{x}_{\mathrm{t}}^{q}-\boldsymbol{x}\right\|_{2}\left(1+\frac{\kappa}{2}\left(1-\frac{3}{\lambda}\right)\right), \\
\geq\left\|\boldsymbol{x}_{\mathrm{t}}^{q}-\boldsymbol{x}\right\|_{2}+\gamma \frac{\left\langle\boldsymbol{x}_{\mathrm{t}}^{q}-\boldsymbol{x}, \boldsymbol{v}\right\rangle}{\left\|\boldsymbol{x}_{\mathrm{t}}^{q}-\boldsymbol{x}\right\|_{2}}\left(1-\frac{3}{\lambda}\right) \\
\geq\left\|\boldsymbol{x}_{\mathrm{t}}^{q}-\boldsymbol{x}\right\|_{2}+\gamma \frac{\left\langle\boldsymbol{x}_{\mathrm{t}}^{q}-\boldsymbol{x}, \boldsymbol{v}\right\rangle}{\left\|\boldsymbol{x}_{\mathrm{t}}^{q}-\boldsymbol{x}\right\|_{2}}-\frac{3 \gamma\|\boldsymbol{v}\|_{2}}{\lambda} .
\end{array}
$$

Using the definition (6), and adding to the bounds above similar bounds on $\left\|\boldsymbol{x}_{\mathrm{s}}^{q}-\tilde{\boldsymbol{x}}\right\|_{2}$, we find from 21]

$$
\left|r^{q}(\tilde{\boldsymbol{x}})-\left(r^{q}(\boldsymbol{x})+\gamma v^{q}(\boldsymbol{x}, \boldsymbol{v})\right)\right|=O\left(\frac{\gamma\|\boldsymbol{v}\|_{2}}{\lambda}\right)=O\left(\frac{\mathrm{c}}{\lambda B}\right) .
$$

Finally, since $\left|\rho_{\boldsymbol{x}, \boldsymbol{v}}^{q}-\rho_{\tilde{\boldsymbol{x}}, \mathbf{0}}^{q}\right| \leq\left|\angle \rho_{\boldsymbol{x}, \boldsymbol{v}}^{q}-\angle \rho_{\tilde{\boldsymbol{x}}, \mathbf{0}}^{q}\right|$, we get $\mid \rho_{\boldsymbol{x}, \boldsymbol{v}}^{q}\left[m_{s}\right]-$ $\rho_{\tilde{\boldsymbol{x}}, \mathbf{0}}^{q}\left[m_{s}\right]\left|\leq \frac{2 \pi m_{s} B}{c M_{s}}\right| r^{q}(\tilde{\boldsymbol{x}})-\left(r^{q}(\boldsymbol{x})+\gamma v^{q}(\boldsymbol{x}, \boldsymbol{v})\right) \mid=O\left(\frac{\pi}{\lambda}\right)$, by using 21] and $\frac{M_{s}}{B} f_{0} T_{s}=\gamma$. This concludes the proof. 


\section{B References}

[1] T.E. Derham, S. Doughty, K. Woodbridge, and C.J. Baker. Design and evaluation of a low-cost multistatic netted radar system. IET Radar Sonar Navig, 5(1):362368, 2007.

[2] T.E. Derham, S. Doughty, C.J. Baker, and K. Woodbridge. Ambiguity functions for spatially coherent and incoherent multistatic radar. IEEE Transactions on aerospace and electronic systems, 46(1):362368, 2010.

[3] A. M. Haimovich, R. S. Blum, and L. J. Cimini. Mimo radar with widely separated antennas. IEEE Signal Processessing Magazine, 25:116129, 2008.

[4] R. Tharmarasa A. A. Gorji and T. Kirubarajan. Widely separated mimo versus multistatic radars for target localization and tracking. IEEE Transactions on Aerospace and Electronics, 49(4):2179-2194, 2013.

[5] F. Gini P. Stinco, M. S. Greco and M. La Manna. Noncooperative target recognition in multistatic radar systems. Radar, Sonar and Navigation, IET, 8(4):396405, 2013.

[6] M.R. McClure and L. Carin. Matching pursuits with a wavebased dictionary. IEEE Transactions On Signal Processing, 45(12):2912-2927, 1997.

[7] E. Hanle. Survey of bistatic and multistatic radar. IEE Proceedings, 133(7):587 - 595, 1986.

[8] M.A. Herman and T. Strohmer. High-resolution radar via compressed sensing. IEEE Transaction Signal Processing, 57(6):2275-2284, 2009.

[9] E.J. Candès and M.B. Wakin. An introduction to compressive sampling. IEEE Signal Processing Magazine, 25(2):21-30, 2008.

[10] M.A. Hadi, S. Alshebeili, K. Jamil, and F.E.A. El-Samie. Compressive sensing applied to radar systems: an overview. Signal, Image and Video Processing, 9(4), 2015.

[11] L. Zheng and X. Wang. Super-resolution delay-doppler estimation for ofdm passive radar. IEEE Transactions on Signal Processing, PP, 102016.

[12] R. Heckel, V. I. Morgenshtern, and M. Soltanolkotabi. Superresolution radar. Information and Inference: A Journal of the IMA, 5(1):22-75, 2016.

[13] S. Gogineni and A. Nehorai. Target estimation using sparse modeling for distributed mimo radar. IEEE Transaction on Signal Processing, 59:5315 - 5325, 2011.

[14] E. Handle. Survey of bistatic and multistatic radars. IEE Proceedings, 133(7), 1987.

[15] F. Ahmad and M.G. Amin. Noncoherent approach to through-the-wall radar localization. IEEE transactions on aerospace and electronic systems, 42(4):7 pp., May 2006.

[16] C.R. Berger and J.M.F. Moura. Noncoherent compressive sensing with application to distributed radar. Annual Conference on Information Sciences and Systems, 45(4):1-6, 2011.

[17] T. Yu, Z. Gong, and B. De. Joint sparse modeling for target parameter estimation in distributed mimo radar. IET International Radar Conference, 2013:719-719, 2013.

[18] B. Sun, H. Chen, X. Wei, and X. Li. Multitarget direct localization using block sparse bayesian learning in distributed mimo radar. International Journal of Antennas and Propagation, 2015:11-23, May 2015.

[19] S.G. Mallat and Z. Zhang. Matching pursuits with timefrequency dictionaries. Signal Processing, IEEE Transactions, 41(12):3397-3415, 1993.

[20] Y.C. Eldar, P. Kuppinger, and H. Bölcskei. Block-sparse signals: Uncertainty relations and efficient recovery. IEEE Transaction on Signal Processing, 58(6):3042 - 3054, 2010.
[21] B. Li and A.P. Petropulu. Distributed mimo radar based on sparse sensing: Analysis and efficient implementation. IEEE Transactions on Aerospace and Electronics Systems, 51(4):3055-3070, 2015.

[22] S. Saponara and B. Neri. Radar sensor signal acquisition and $3 \mathrm{~d} \mathrm{fft}$ processing for smart mobility surveillance systems. 2016 IEEE Sensors Applications Symposium (SAS), pages 16, 2016.

[23] W. C.Hsu J.J. Lin, Y.P. Li and T.S. Lee. Design of an fmcw radar baseband signal processing system for automotive application. Leuzzi F., Ferilli S. (eds) Traffic Mining Applied to Police Activities. TRAP 2017. Advances in Intelligent Systems and Computing, 5(42), 2016.

[24] F. Cuccoli S. Capobianco, L. Facheris and S. Marinai. Vehicle classification based onconvolutional networks applied tofmcw radar signals. Leuzzi F., Ferilli S. (eds) Traffic Mining Applied to Police Activities. TRAP 2017. Advances in Intelligent Systems and Computing, 728, 2018.

[25] S. H. Dokhanchi, B. S. Mysore, K. V. Mishra, and B. Ottersten. A mmwave automotive joint radar-communications system. IEEE Transactions on Aerospace and Electronic Systems, 55(3):1241-1260, June 2019.

[26] A. Abtahi, S. Gazor, and F. Marvasti. Off-grid localization in mimo radars using sparsity. IEEE Signal Processing Letters, PP(99):1-1, 2018.

[27] Y. Liu, H. Meng, G. Li, and X. Wang. Velocity estimation and range shift compensation for high range resolution profiling in stepped-frequency radar. Geoscience and Remote Sensing Letters, IEEE, 7:791 - 795, 112010.

[28] H. Bao. The research of velocity compensation method based on range-profile function. International Journal of Hybrid Information Technology, 7:49-56, 032014.

[29] T. Feuillen, A. Mallat, and L. Vandendorpe. Stepped frequency radar for automotive application: Range-doppler coupling and distortions analysis. In MILCOM 2016 - 2016 IEEE Military Communications Conference, pages 894-899, Nov 2016.

[30] J.A. Tropp. Just relax: Convex programming methods for subset selection and sparse approximation. IEEE Transactions on Information Theory - TIT, 04-04, 012004.

[31] Y.C. Pati, R. Rezaiifar, and P.S. Krishnaprasad. Orthogonal matching pursuit: recursive function approximation with application to wavelet decomposition. Asilomar Conf. On Signals, Systems and Comput, page 4044, 1995.

[32] S. Foucart and H. Rauhut. A Mathematical Introduction to Compressive Sensing. 2013.

[33] R. Gribonval. Fast matching pursuit with a multiscale dictionary of gaussian chirps. IEEE Transactions on Signal Processing, 49(5):4044, May 2001.

[34] I.M. Ivashko, O.A. Krasnov, and A.G. Yarovoy. Sparsitybased optimization of the sensors positions in radar networks with separated transmit and receive nodes. International Journal of Distributed Sensor Networks, 2016, 2016.

[35] I.M. Ivashko, G. Leusa, and A. Yarovoy. Radar network topology optimization for joint target position and velocity estimation. Signal Processing, 130, 2016.

[36] D.W. O' Hagan, S.R. Doughty, and M.R. Inggs. Academic Press Library in Signal Processing, volume 7. 2018. 